\title{
FACE RECOGNITION APPROACH BASED ON WAVELET - CURVELET TECHNIQUE
}

\author{
Muzhir Shaban Al-Ani1 and Alaa Sulaiman Al-waisy2 \\ Department of Computer Science, College of Computer, Al-Anbar University, Iraq. \\ muzhir_shaban@yahoo.com king_alaa87@yahoo.com
}

\begin{abstract}
In this paper, a novel face recognition approach based on wavelet-curvelet technique, is proposed. This algorithm based on the similarities embedded in the images, That utilize the wavelet-curvelet technique to extract facial features. The implemented technique can overcome on the other mathematical image analysis approaches. This approaches may suffered from the potential for a high dimensional feature space, Therefore it aims to reduce the dimensionality that reduce the required computational power and memory size. Then the Nearest Mean Classifier (NMC) is adopted to recognize different faces. In this work, three major experiments were done. two face databases $(M A F D \& O R L$, and higher recognition rate is obtained by the implementation of this techniques.
\end{abstract}

\section{KEYWORDS:}

Face recognition, wavelet transform, curvelet transform, Nearest Mean Classifier.

\section{INTRODUCTION}

Pattern recognition systems have been designed to classify many different kinds of patterns such as face recognition, fingerprint recognition, iris recognition, medical diagnosis of patients from the experimental results and several other applications[1]. Utilizing biometrics for personal authentication is becoming more accurate than current methods (such as the utilization of passwords, keys or identification cards) and more convenient because there is nothing to carry or remember and a biometric trait cannot be easily (transferred or duplicated) and lost [2,3]. The main focus of this paper on the face recognition. It is a pattern recognition task performed specifically on face images. It can be described as classifying a face either "known" or "unknown", after comparing it with previously stored information from various faces[4]. Face recognition presents a challenging problem in the field of in pattern recognition, image \& signal processing and computer vision. It is also gained considerable attention over recent decades because of its many applications in various fields [5].

Biometric recognition systems based on face recognition have shown excellent performance in the area of secured to buildings/ airports/seaports, border checkpoints, law enforcement, surveillance systems and so on. Face recognition problem is very challenging because of variations in different face images of the same person due to changes in facial expressions, multiposes, illumination conditions, rotation, age, and presence of beard, moustache and etc $[4,5,6]$. Therefore developing a computational model of face recognition is quite difficult, because faces are very complex. In general, a face recognition system involves three important stages: Face detection, Feature extraction and (identification and/or verification). The robustness of face 
recognition could be improved by treating the variations in these stages. Feature extraction is a very important step for face recognition. Which reduces the number of features, removes irrelevant, noisy and redundant data, and results in acceptable recognition accuracy.

In this paper, novel feature extraction method, named as wavelet-curvelet technique, is implemented. Based on the similarities embedded in the images, The proposed technique can overcome on the other mathematical image analysis approaches. Which can be suffered from the potential for a high dimensional feature space. Therefore the proposed technique aims to reduce the dimensionality to reduce the required computational power and memory consumption. Then the Nearest Mean Classifier (NMC) is adopted to recognize different faces. In this work, three major experiments were done. two face databases (MAFD \& ORL, and higher recognition rate is obtained by the implementation of this techniques. This paper is organized into the following sections. Necessary background for the transformation methods (wavelet and curvelet) are described in section 2. Overview of the related work is given in section 3. The implemented face recognition method is presented in Section 4. Some comparative test results are reported in Section 5. The paper is concluded in Section 6.

\section{Transformation MethodS}

The transformation is a process that transforms an object from a given domain to another which can be used for its recognition. A large class of image processing transformations is linear in nature an output image is formed from linear combinations of pixels of an input image [7]. This section explains some typical examples of linear transforms.

\subsection{Wavelet Transform}

The Wavelet Transform was introduced at the beginning of the 1980s by Morlet, who used it to evaluate seismic data. Wavelet transform provide an alternative to classical Fourier methods for one and multi-dimensional data analysis and synthesis [8].Wavelet transform has been widely applied to engineering fields, including signal and image processing, geophysical signal processing, computer vision and encoding, speech synthesis and analysis, signal singularity detection and spectrum estimation, pattern recognition, fractal and chaos theory, quantum physics and etc [9].

The wavelets are functions defined over a finite interval. The basic idea of the wavelet transform is to represent an arbitrary function $f(\mathrm{x})$ as a linear combination of a set of wavelets or basis functions. The purpose of wavelet transform is to change the data from time-space domain to time frequency domain which is useful in digital signal processing and image compression. In signal processing wavelet is helpful to recover weak signals from noise. But in image compression wavelet have been used to compress images to an extent greater than it is generally possible with the other methods [10]. The Discrete Wavelet Transform (DWT) is a special case of the wavelet transform that provides a compact representation of a signal in time and frequency that can be efficiently computed [11]. In the two-band multi-resolution wavelet transform, signal can be expressed by wavelet and scaling basis functions at different scale, in a hierarchical manner.

$$
f(x)=\sum_{k} \alpha_{0, k} \phi_{0, k}(x)+\sum_{j} \sum_{k} d_{j, k} \psi_{j, k}(x) \ldots \ldots(1)
$$

$\emptyset_{\mathbf{j}, \mathbf{k}}$ are scaling functions at scale $\mathbf{j}$, and $\psi_{\mathbf{j}, \mathbf{k}}$ are wavelet functions at scale $\mathrm{j} \alpha_{\mathbf{j}, \mathbf{k}}$, and $\mathrm{d}_{\mathbf{j}, \mathbf{k}}$ are scaling coefficients and wavelet coefficients. 
Signal \& Image Processing : An International Journal (SIPIJ) Vol.3, No.2, April 2012

\subsection{Curvelet Transform}

To overcome the innate limitations of traditional multi-scale representations such as wavelet a novel transform has been developed by Candes and Donoho in 1999 known as curvelet transform. Curvelet transform is a multiscale pyramid with many directions, positions at each length and fine scales [12]. The motivation for the development of the new transform was to find a way to represent edges and other singularities along curves in a way that was more efficient than existing methods, that is, less coefficients are required to reconstruct an edge to a given degree of accuracy [13].

The curvelet transform, like the wavelet transform, is a multiscale transform, with frame elements indexed by scale and location parameters. Unlike the wavelet transform, it has directional parameters, and the curvelet pyramid contains elements with a very high degree of directional specificity. Also, the curvelet transform is based on a certain anisotropic scaling principle which is quite different from the isotropic scaling of wavelets. In wavelet's isotropic principle, the length and width of support frame is of equal size whereas in curvelet transform the width and length are related by the relation width $\approx$ length 2 that is known as parabolic or anisotropic scaling [12]. There are two generations of the curvelet transform. The idea of The First Generation Discrete Curvelet Transform (DCTG1) is first to decompose the image into a set of wavelet bands, and analyze each band by a local ridgelet transform. It results in a large amount of redundancy. Moreover, this process is very time consuming, which makes it less feasible for facial features analysis in a large database [14].

To overcome on the all the drawbacks in the (DCTG1) such as the parabolic scaling ratio width $\approx$ length 2 is not completely true and time consuming. The Second Generation Curvelet Transform (DCTG2) introduced in 2006 is not only simpler, but is faster and less redundant compared to its first generation version [15]. Currently two implementations of fast (DCTG2) are available i.e. Unequally-Spaced Fast Fourier Transform (USFFT) Based Curvelet and Frequency Wrapping Based Curvelet. The difference is the choice of spatial grid used to translate curvelet at each scale and angle [12].

\section{LITERATURE SURVEY}

Automatic face recognition has attracted many researchers and scientists and as a consequence, several techniques have been developed to solve this problem. Amongst all these numerous techniques very few are capable of solving the problem in unconstrained environment. Generally, Several researchers in the field of face recognition developed different recognition methods. Some of these researches are summarized below:

Ming Li , Fuwen $\mathrm{Wu}$ and Xueyan Liu presented a face recognition algorithm which uses wavelet transform to obtain on low frequency approximation subband. Then the Fast Independent Component Analysis (FastICA) is applied to extract features from the approximation subband. finally, the extracted features are classified through Radial Basis Function (RBF) neural networks [16].

$\mathrm{Yu} \mathrm{Su}$,Shiguang Shan and et al proposed a face recognition method which based on both global and local discriminative features. In this method, global features are extracted from the whole face images by keeping the low-frequency coefficients of 2D-discrete fourier transform which is believe encodes the holistic facial information such as eyes, mouth and the whole facial contour. For local feature extraction, gabor wavelets are used considering their biological relevance. After that, Fisher's Linear Discriminant (FLD) is separately applied to the global Fourier features and each local patch of Gabor features. Thus, multiple FLD classifiers are obtained, each embodying 
Signal \& Image Processing : An International Journal (SIPIJ) Vol.3, No.2, April 2012

different facial evidences for face recognition. Finally, all these classifiers are combined to form a Hierarchical Ensemble Classifier (HEC) [17].

Niu Liping, Li XinYuan and et al presented a hybrid approach based on Bayesian and wavelet transform for face recognition is proposed. Firstly the system uses (PCA) to select the first 10 candidate images. Then these candidate images and each testing image are decomposed into low frequency and high frequency sub-band images by applying wavelet transform. Finally Bayesian recognition is parallel processed using these sub-band images [18].

Mohammed Rziza, Mohamed El .Aroussi and et al in this work, an efficient local appearance feature extraction method based the Curvelet Transform (CT) is proposed in order to further enhance the performance of the well known Linear Discriminant Analysis (LDA) method when applied to face recognition [19].

Dinesh Kumar, Shakti Kumar and et al presented a PCA-Memetic Algorithm (PCA-MA) approach for feature selection. The ( PCA) has been extended by MAs where the former was used for feature extraction/dimensionality reduction and the latter exploited for feature selection. Simulations were performed over ORL and YaleB face databases using Euclidean norm as the classifier. The same approach has also been applied to (LDA) and Kernel PCA approaches with the MA [20].

\section{IMPLEMENTED APPROACH}

The implemented Approach can be summarized in the three steps as shown in the figure (1). Wavelet transform, Curvelet transform and Nearest Mean Classifier (NMC).

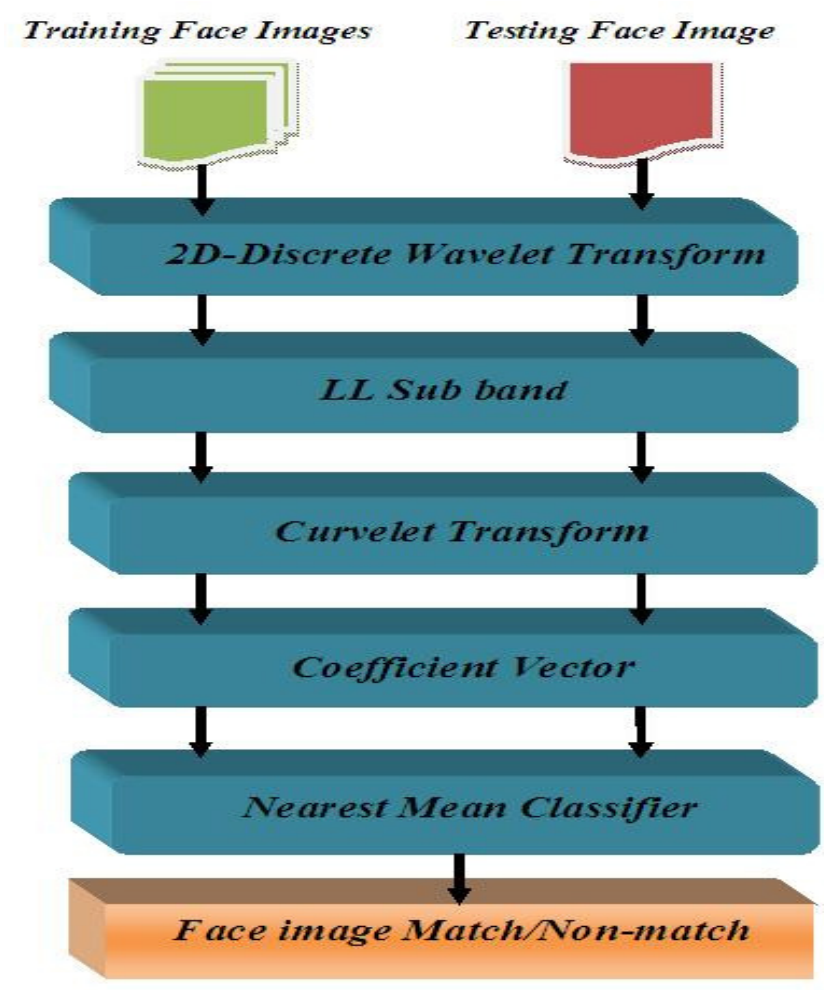

Figure (1) : The implemented face recognition approach 


\subsection{D-Discrete Wavelet Transform (2D_DWT)}

In the implemented technique two levels of the 2D-DWT is used to decompose the face image into a multiresolution representation in order to keep the least coefficients possible without losing useful image information. In the implemented system the Daubechies-2 wavelet type is used with two types of filters ( Low_pass \& High_pass filters).Typical wavelet decomposition can be written as:

$$
f_{n}(x, y)=f_{n-1}(x, y)+g_{n-1}(x, y) \ldots \ldots \ldots \ldots \ldots(2)
$$

Where,

$$
g_{n-1}=g_{L H, n-1}(x, y)+g_{H L, n-1}(x, y)+g_{H H, n-1}(x, y) \ldots \ldots \ldots(3)
$$

The above equations indicate that any image signal can be decomposed into a specific wavelet domain. Where the subscripts $\mathrm{L}$ and $\mathrm{H}$ are used to indicate low and high frequency components. Here we adopt a recursive algorithm to compute these low and high frequency components.

$$
\begin{aligned}
& A_{L i, k 1, k 2}^{(n-1)}=\sum_{n, l 2} a_{l 1-2 k 1, \alpha_{l 2-2 k 2}} A_{L L, 11, n 2}^{(n)} \ldots(4)
\end{aligned}
$$

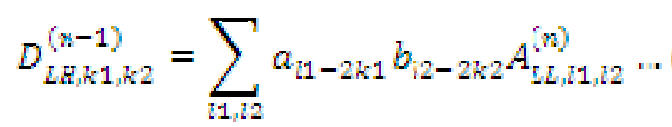

$$
\begin{aligned}
& D_{H, k 1, k 2}^{(x-1)}=\sum_{i 1, n 2} b_{l 1-2 k 1} a_{i 2-2 k 2} A_{[L, 11,2}^{m)} \ldots(6) \\
& D_{H H, k 1, k 2}^{(n-1)}=\sum_{l 1,2} b_{l 1-2 k 1} b_{l 2-2 k 2} A_{L, i, l, l 2}^{(n)} \cdots(7)
\end{aligned}
$$

where $\left\{a_{k}\right\}$ and $\left\{b_{k}\right\}$ are filter decomposition sequences related to scale function $\varphi(t)$ and wavelet function $\psi(t)$, which result in various wavelet transformation, such as Daubechies. At each decomposition level, the low-pass filter produces a sub-image $A_{L L, k 1, k 2}^{(n-1)}$ which consists of only half wavelet coefficients of those inputted to the filter, whereas keeping most of the input information. The outputs of the high-pass filters $D_{L H, k 1, k 2}^{(n-1)}, D_{H L, k 1, k 2}^{(n-1)}$ and $D_{H H, k 1, k 2}^{(n-1)}$ are three sub-images with the same size as low-pass sub-image, which can present different image details in different directions. In the implemented recognition schema only the approximation subband is used as the input to the next step, which believe encodes the holistic facial information such as eyes, mouth and the whole facial contour. Figure(2) (a) demonstrates the decomposition process by applying (2-DWT) of a face image in level1 and figure(2) (b) depicts two levels wavelet decomposition by applying wavelet transform on the low-frequency band sequentially. 
Signal \& Image Processing : An International Journal (SIPIJ) Vol.3, No.2, April 2012

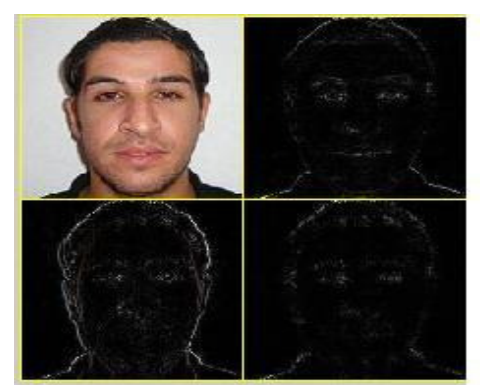

a) 1-level wavelet decomposition

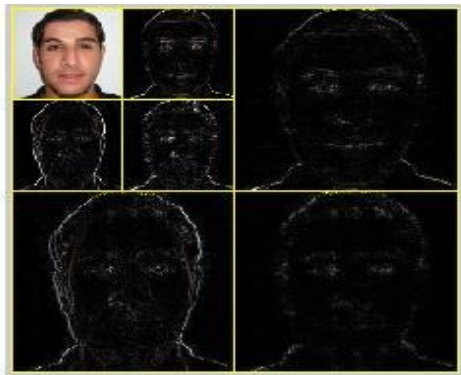

b) 2-level wavelet decomposition

Figure(2): Face image in wavelet subbands

\subsection{Curvelet Via Wrapping}

In the implemented algorithm the Curvelet Via Wrapping is applied on the approximation subband of the (2D-DWT). Wrapping based curvelet transform is faster in computation time and more robust than ridgelet and (USFFT) based curvelet transform. Curvelet transform based on wrapping of Fourier samples takes a 2-D image as input in the form of a Cartesian array $\mathrm{f}[\mathrm{n} 1, \mathrm{n} 2]$ such that $0 \leq \mathrm{n} 1, \mathrm{n} 2<\mathrm{N}$ and generates a number of curvelet coefficients indexed by a scale $\mathrm{j}$, an orientation 1 and two spatial location parameters $(\mathrm{k} 1, \mathrm{k} 2)$ as output. The discrete curvelet transform can be implemented based on the wrapping algorithm. In this algorithm, four steps are carried out:

1- The 2D-Img is first transformed into the frequency domain by forward FFT and obtain Fourier samples $f[n 1, n 2]$.

2- for each scale $\mathrm{j}$ and angle $1 \mathrm{do}$

Divide FFT into collection of Digital Corona Tiles (Wedges) by using two windowing functions 'radial window' and 'angular window'. As explained in the following figure (3).

Move the tile to the origin point. figure (4)(a).

3- Apply the wrapping algorithm to the wedge data. figure (4)(b).

4- Apply the inverse 2D FFT to the wrapped data to get curvelet coefficients. figure (5).

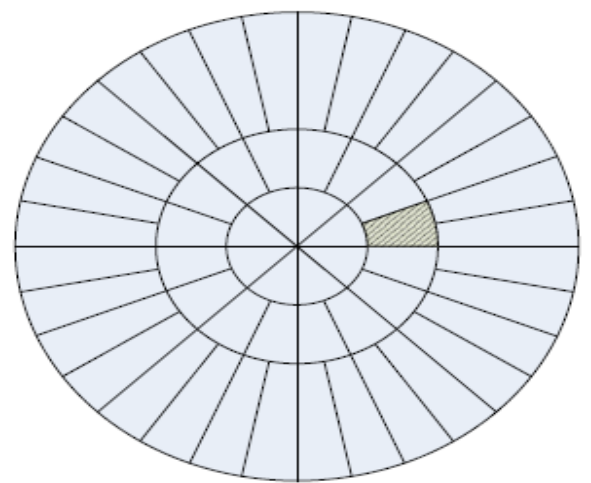

Figure (3): Digital Corona tiling of the Frequency Domain. 


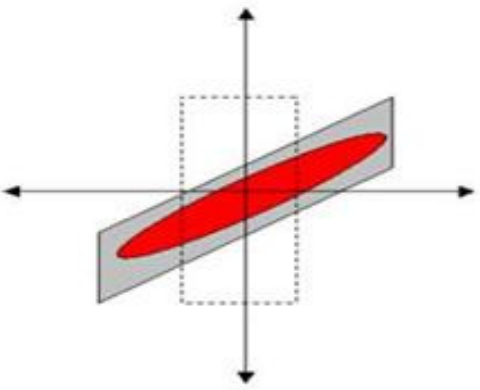

(a) Before wrapping

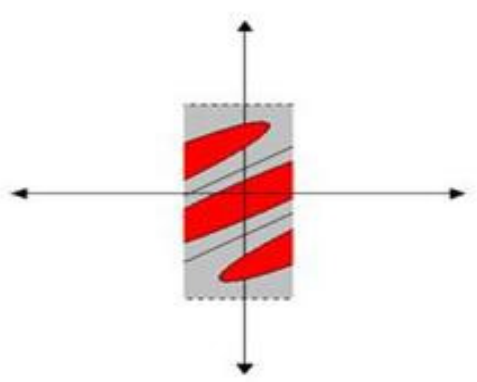

(b) After wrapping

Figure (4): Curvelet Via Wrapping.

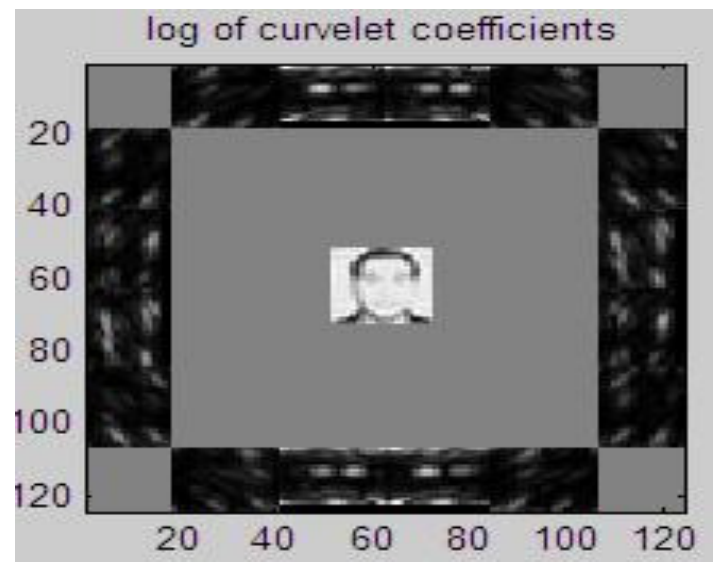

Figure (5): Curvelet coefficients.

\subsection{Nearest Mean Classifier}

The classification step is implemented based on the Nearest Mean Classifier (NMC). In the NMC, the Euclidean distance from each class mean (in this case) is computed for the decision of the class of the training sample and the test data. In mathematical terms, the Euclidean Distance between the test sample $A_{p}$ and any one of the training sample $B_{p}^{Q}$

$$
\left.D(q)=\frac{\sum_{m} \Sigma_{n}\left(A_{p}-\bar{A}_{p}\right)\left(B_{p}^{q}-\overline{B_{p}^{q}}\right)}{\sqrt{\left(\sum_{m} \Sigma_{n}\left(A_{p}-\bar{A}_{p}\right)^{2}\right)\left(\Sigma_{m} \Sigma_{n}\left(B_{p}^{n_{p}}-\overline{B_{p}^{q}}\right)^{2}\right)}} \ldots \ldots . . .8\right)
$$

Where $m$ and $n$ is the dimensions of sample and $\bar{A}_{p}$ and $\bar{B}_{p}^{Q}$ is the mean value of the testing and training samples, respectively. After computing the distance to each class if the testing image as the same as the training image then the $\mathrm{D}(\mathrm{q})$ is equal to one else the return value between $(\mathrm{o}, 1)$. 


\section{EXPERIMENTAL RESULTS}

The implemented face recognition algorithm is tested on the two face databases. Considering the number of the training and testing images to be 5 in the two cases. Firstly, the experiment was done by constructing multi-view face database named as Muzhir_Alaa Face Database (MAFD). This database currently contains colored face images of 50 persons. For each person, we take 10 photographs. Each photograph has a different combination of viewpoint such as (frontal $0^{\circ}$, right $45^{\circ}$, right $90^{\circ}$, left $45^{\circ}$ and left $90^{\circ}$ ) and facial expression such as smiling, laughing, neutral and closed eyes).The image size is ( $256 \times 256)$. Figure (6) shows the image variations for three persons.

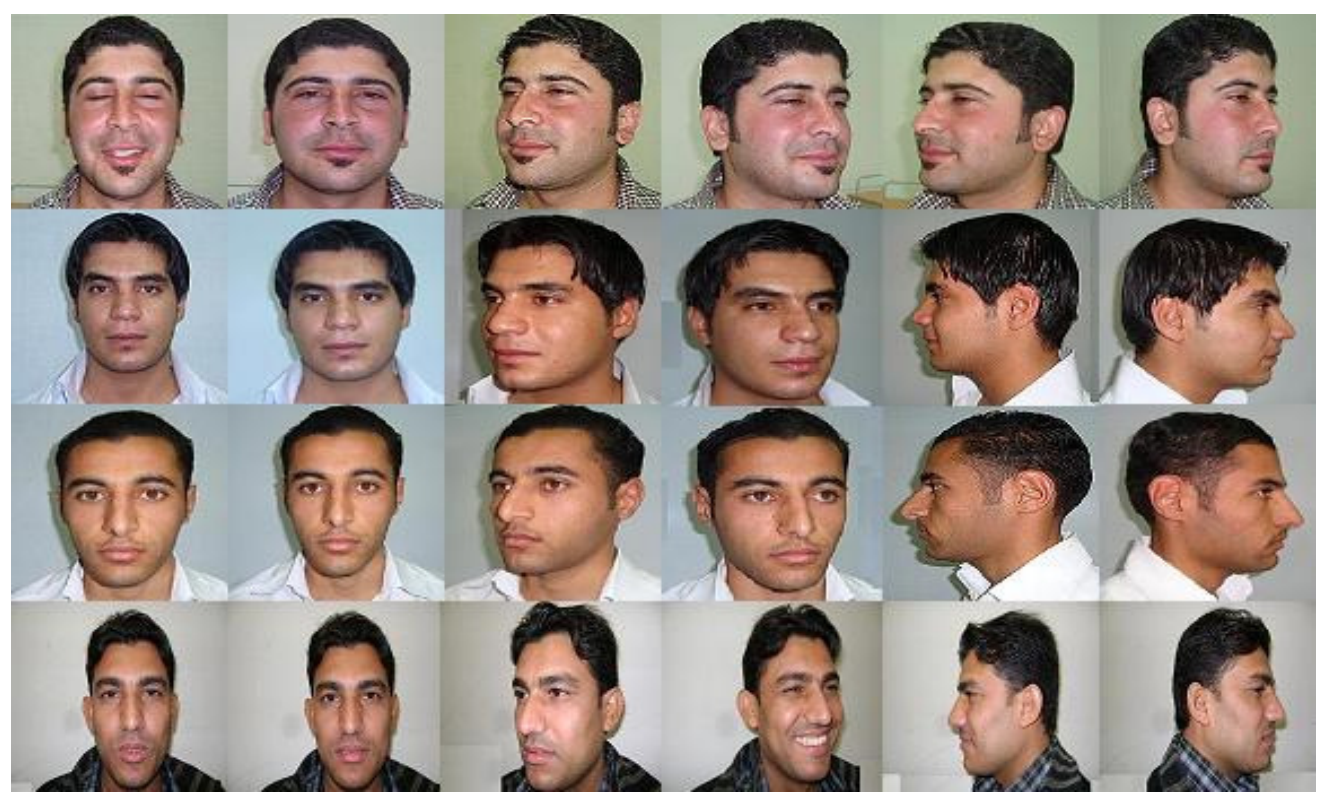

Figure(6): Image variation of some persons in the constructed face database

The Second face recognition experiment is carried out on ORL database, with 40 persons each one has 10 face images were taken at different times, which contain quite a high degree of variability in illumination conditions, facial expressions and multi-views thus it is appropriate to test run the algorithm. The face recognition rate of the implemented method in the above two experiments are explained in the figure (7). The training time and testing time of the first experiment are summarized in table 1 . Every technique in the implemented face recognition algorithm used 250 images during training phase. The training time and testing time of the second experiment are summarized in table 2 . Every technique in the implemented face recognition algorithm used 200 images during training phase. The testing time is calculated for each image. 
Signal \& Image Processing : An International Journal (SIPIJ) Vol.3, No.2, April 2012

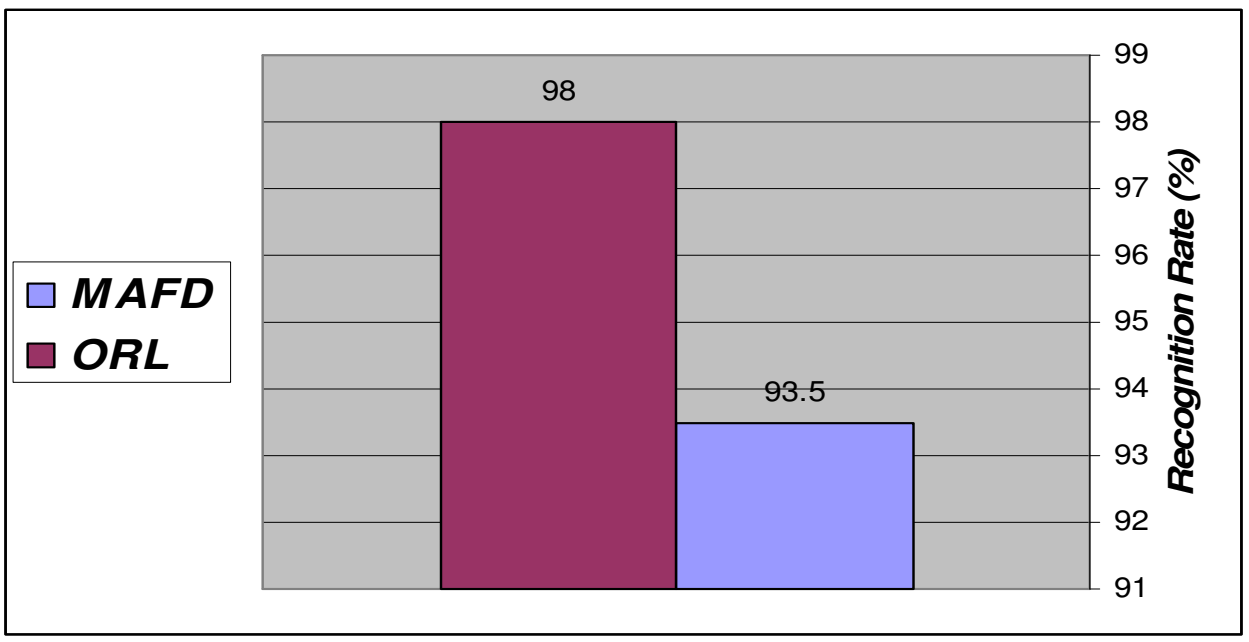

Figure (7): Recognition accuracy of the implemented algorithm

Table 1. The training time and testing time of MAFD

\begin{tabular}{|c|c|c|}
\hline The Method & Training Time $(s)$ & Testing Time $(s)$ \\
\hline Wavelet & 11.711305 & 4.046338 \\
\hline Curvelet & 176.347929 & 4.968537 \\
\hline Wavelet-Curvelet & 29.362749 & 3.744976 \\
\hline
\end{tabular}

Table 2. The training time and testing time of ORL

\begin{tabular}{|c|c|c|}
\hline The Method & Training Time $(s)$ & Testing Time $(s)$ \\
\hline Wavelet & 3.367464 & 3.626934 \\
\hline Curvelet & 30.967317 & 3.597878 \\
\hline Wavelet-Curvelet & 7.230028 & 3.731235 \\
\hline
\end{tabular}

\section{Conclusions}

In this paper, a novel feature extraction approach via wavelet-curvelet technique is implemented to extract the facial features and applied it to face recognition. This approach based on the similarities preserved by wavelet and curvelet transform. The implemented technique can overcome on the other mathematical image analysis approaches. The implemented technique aims to reduce the dimensionality to reduce the required computational power and memory consumption. An important observation must be explained here, that when the curvelet transform was applied individually on the image of size $(256 \times 256)$ it takes longest execution time but when combined it with wavelet transform the execution time is lower because of the output of the wavelet transform is an image of size (64 x 64) this leads to lower dimensionality and reduce the required computational power. Then the Nearest Mean Classifier (NMC) is adopted to recognize different faces. Extensive comparison tests on two face databases are carried out. Firstly, the experiment was done by constructing multi-view face database named as (MAFD) and the obtained recognition rate equal to $93.6 \%$. The Second face recognition experiment is carried out on ORL database. the obtained high recognition rate equal to $98 \%$. 
Signal \& Image Processing : An International Journal (SIPIJ) Vol.3, No.2, April 2012

\section{REFERENCES}

[1] Mohammad Shahin Mahanta, " Linear Feature Extraction with Emphasis on Face Recognition" , Graduate Department of Electrical and Computer Engineering University of Toronto, Copyright (C) 2009

[2] Dr. Salah M. Rahal, Dr. Hatim A. Abu Samah and et al," Secure Identification System - SIS", College of Computer \& Information Sciences, King Saud University - 2006.

[3] Sarat C. Dass Anil K. Jain, " Fingerprint-Based Recognition", September 20, 2006.

[4] Yusuf Atilgan, " FACE RECOGNITION", MAY, 2009.

[5] Rabia Jafri* and Hamid R. Arabnia*," A Survey of Face Recognition Techniques", Journal of Information Processing Systems, Vol.5, No.2, June 2009.

[6] Anil K. Jain, Arun Ross and Salil Prabhakar, " An Introduction to Biometric Recognition", IEEE Transactions On Circuits And Systems For Video Technology, VOL. 14, NO. 1, JANUARY 2004.

[7] Gerhard X. Ritter and Joseph N. Wilson, " Handbook of Computer Vision Algorithms in Image Algebra", ISBN: 0849326362 Pub Date: 05/01/96.

[8] Ali Karami *, Bahman Zanj and Azadeh Kiani Sarkaleh, " Persian sign language (PSL) recognition using wavelet transform and neural networks", Faculty of Engineering, University of Guilan, P.O. Box 41635-3756, Rasht, Iran, journal homepage: www.elsevier.com/locate/eswa, 3 September 2010.

[9] Zhao Lihong, Song Ying, Zhu Yushi, Zhang Cheng, Zhang Xili, " Face Recognition Based on Image Transformation", 978-0-7695-3571-5/09 \$25.00 @ 2009 IEEE .

[10] Aunss Sinan Maki, " Hand Palm Recognition Using Wavelet Transform", Al-Nahrain University College of Science, 2004.

[11] Harin Sellahewa and Sabah A. Jassim, "Image-Quality-Based Adaptive Face Recognition", Ieee Transactions On Instrumentation And Measurement, Vol. 59, NO. 4, APRIL 2010.

[12] SHREEJA R and SHALINI BHATIA, " Facial Feature Extraction Using Statistical Quantities Of Curve Coefficients", International Journal of Engineering Science and Technology Vol. 2(10), 2010.

[13] Rowan Seymour, Darryl Stewart and JiMing, " Comparison of Image Transform-Based Features for Visual Speech Recognition in Clean and Corrupted Videos", EURASIP Journal on Image and Video Processing, Volume 2008.

[14] Ishrat Jahan Sumana, " Image Retrieval Using Discrete Curvelet Transform", Monash University, Australia, November, 2008.

[15] Jianhong Xie, " Face Recognition Based on Curvelet Transform and LS-SVM", Proceedings of the 2009 International Symposium on Information Processing (ISIP’09), Huangshan, P. R. China, August 21-23, 2009, pp. 140-143.

[16] Ming Li and Fuwen Wu, Xueyan Liu, " Face Recognition Based on WT, FastICA and RBF Neural Network", Third International Conference on Natural Computation 0-7695-2875-9/07 \$25.00 (C) 2007 IEEE.

[17] Yu Su, Shiguang Shan, Xilin Chen and Wen Gao, " Hierarchical Ensemble of Global and Local Classifiers for Face Recognition", IEEE Transactions On Image Processing, VOL. 18, NO. 8, AUGUST 2009.

[18] Niu Liping, Li XinYuan and Dou Yuqiang, " Bayesian Face Recognition Using Wavelet Transform", 978-0-7695-3752-8/09 \$25.00 @ 2009 IEEE.

[19] Mohammed Rziza, Mohamed El Aroussi, Mohammed El Hassouni, Sanaa Ghouzali and Driss Aboutajdine," Local Curvelet Based Classification Using Linear Discriminant Analysis for Face Recognition", International Journal of Computer Science 4:1 2009.

[20] Dinesh KUMAR, Shakti KUMAR and C. S. RAI, " Feature selection for face recognition: a memetic algorithmic approach", Journal of Zhejiang University SCIENCE, June 10, 2009. 
Signal \& Image Processing : An International Journal (SIPIJ) Vol.3, No.2, April 2012

\section{Authors}

${ }^{1}$ Muzhir Shaban Al-Ani has received $\mathrm{Ph}$. D. in Computer \& Communication Engineering Technology, ETSII, Valladolid University, Spain, 1994. Assistant of Dean at Al-Anbar Technical Institute (1985). Head of Electrical Department at Al-Anbar Technical Institute, Iraq (19851988), Head of Computer and Software Engineering Department at AlMustansyria University, Iraq (1997-2001), Dean of Computer Science (CS) \& Information System (IS) faculty at University of Technology, Iraq (20012003). He joined in 15 September 2003 Electrical and Computer Engineering Department, College of Engineering, Applied Science University, Amman, Jordan, as Associated Professor. He joined in 15 September 2005 Management Information System Department, Amman Arab University, Amman, Jordan, as Associated Professor, then he joined computer science department in 15 September 2008 at the same university.

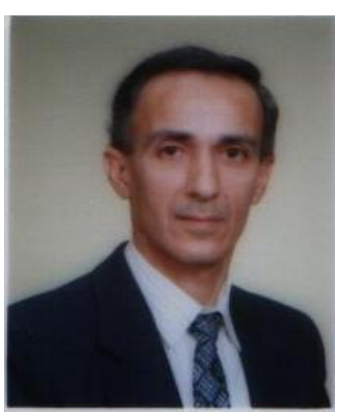

${ }^{2}$ Alaa Sulaiman Nassar Al-Waisy has received B.Sc in Computer Science, Al-Anbar University, Iraq, (2005-2009). M.Sc student (2010- tell now) in Computer Science Department, Al-nabar University. Fields of interest: computer vision, image processing, face recognition, face detection and related fields. Al-Waisy taught many subjects such as operation system, cryptography and encryption.

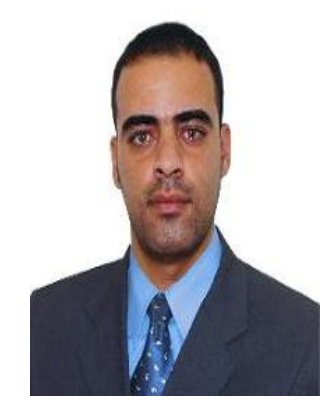

